OPEN ACCESS

Edited by:

San-Gang Wu,

First Affiliated Hospital of Xiamen

University, China

Reviewed by:

Nian-Sheng Tzeng,

Tri-Service General Hospital, Taiwan

Manuel Pires Bicho,

University of Lisbon, Portugal

*Correspondence:

Huaidong Cheng

chd1975ay@126.com

${ }^{t}$ These authors have contributed equally to this work

Specialty section: This article was submitted to Breast Cancer,

a section of the journal

Frontiers in Oncology

Received: 17 November 2021 Accepted: 12 January 2022

Published: 08 February 2022

Citation:

Li W, Zhang Q, Cai Y, Chen T and Cheng H (2022) The COMT Genetic Factor Regulates Chemotherapy-

Related Prospective Memory Impairment in Survivors With HER2-/+ Breast Cancer.

Front. Oncol. 12:816923. doi: 10.3389/fonc.2022.816923

\section{The COMT Genetic Factor Regulates Chemotherapy-Related Prospective Memory Impairment in Survivors With HER2-/+ Breast Cancer}

\author{
Wen $\mathrm{Li}^{\dagger}$, Qianqian Zhang ${ }^{\dagger}$, Yinlian $\mathrm{Cai}^{\dagger}$, Tingting Chen and Huaidong Cheng * \\ Cancer Treatment Center, the Second Affiliated Hospital of Anhui Medical University, Hefei, China
}

Background: Previous findings indicated that polymorphism in gene catechol-Omethyltransferase (COMT) had been linked to chemotherapy-related cognitive impairment $(\mathrm{CRCl})$. Nevertheless, the motivation of COMT polymorphisms in regulating cognitive impairment in breast cancer survivors with disparate status of human epidermal growth factor receptor 2 (HER2) was still vague.

Objective: The current research aimed to evaluate the regulation of the risk by COMT genotype on $\mathrm{CRCl}$ in breast cancer survivors with disparate status of HER2.

Methods: Breast cancer survivors (103 with HER2- and 118 with HER2+) underwent neuropsychological tests before and after chemotherapy, containing event- and timebased prospective memory (EBPM and TBPM). Three single-nucleotide polymorphisms (SNPs) were estimated by providing peripheral blood, containing COMT (rs165599, rs737865, and rs4680).

Results: The EBPM and TBPM performances was lower as compared with these before chemotherapy ( $z=-7.712, z=-2.403$, respectively, $p<0.01)$. Furthermore, the EBPM and TBPM performances of HER2- group survivors were lower than those of HER2+ group survivors after chemotherapy $(z=-7.181, p<0.01 ; z=-2.205 p<0.05$, respectively). The survivors with COMT (rs165599) A A genotype carriers had a meaningfully poorer chance of memory descend [dominant model: adjusted, $\mathrm{OR}=2.21, \mathrm{Cl}(95 \%)=1.156-4.225, p=0.016$ ] and showed better on TBPM test, relative to $G / G$ genotype. Patients with the COMT (rs737865) $A / G$ and $G / G$ genotype showed protective function than the patients with the A A and performed better on MMSE and TBPM tests.

Conclusion: The types of HER2 may be correlated to chemotherapy-related prospective memory impairments in breast cancer survivors. Furthermore, the COMT (rs165599, rs737865) polymorphisms were correlated to the risk of TBPM decline scores and possibly be a potential genetic identifying for increasing risk of $\mathrm{CRCl}$ in breast cancer patients with disparate status of HER2.

Keywords: catechol-O-methyltransferase (COMT), polymorphisms, chemotherapy, memory, human epidermal growth factor receptor 2 (HER2), breast cancer 


\section{INTRODUCTION}

Breast cancer is the most familiar malignancy in Chinese women and the sixth main cause of cancer-related death (1). By the end of 2008, 169,452 new breast cancer cases were reported in China; 44,908 cases were related deaths (2). It is reported that 1 in $8-10$ women in the United States will suffer from breast cancer during their lifetime (3). The incidence rate of breast cancer increased by about $0.3 \%$ every year from 2012 to 2016 . On the contrary, the mortality rate decreased year by year, decreasing 40\% from 1989 to 2017 , which avoided the death of 375,900 breast cancer patients (4). Chemotherapy is one of the most main therapeutic methods for breast cancer; the 5-year survival rate of early breast cancer is close to $90 \%$, which leads to a growing concern about the side effects of chemotherapy treatment (5). In addition to the common clinical side effects such as nausea, vomiting, bone marrow suppression, and hair loss, the impact of chemotherapy on cognitive function has attracted more and more attention in the world (6). A large body of evidence have reported that breast cancer patients experience a moderate to severe degree of cognitive impairment during or after chemotherapy (7-9). These cognitive function deficits involved memory, attention, information processing speed, executive function, and visual space function. This phenomenon is referred to as chemotherapy-related cognitive impairment (CRCI) (10). It is estimated that about $35 \%-70 \%$ of breast cancer patients develop CRCI after chemotherapy, which makes survivors unable to recover from pre-cancer life even after the end of treatment, having significant impact on their daily work and life and greatly reducing their quality of life (QOL) (11).

Prospective memory (PM) is outlined as the ability of remembering to carry out a purpose behavior at a convinced time or place in the future. It not only plays an important role in daily life but also an important part of advanced cognitive activities and is a key factor affecting the recovery of patients' cognitive function (12). PM was usually fallen into two groups: event-based PM (EBPM) and time-based PM (TBPM). Our previous studies found that patients with breast cancer had PM impairment after chemotherapy, especially significant deficit in EBPM (9).

Breast cancer is a highly heterogeneous malignancy; the most important research direction was concentrated in the field of molecular typing (13). The gene status of human epidermal growth factor receptor 2 (HER2) is important, which is key clinical-pathological characteristic for the prognosis recovery of breast cancer (14). There is an online comment that the main confusion in the CRCI study of breast cancer is its significant heterogeneity, as published in CA: A Cancer Journal for Clinicians (15). Our previous research findings simulated that heterogeneity among CRCI in breast cancer survivors with estrogen/progesterone receptor negative (ER-/PR-), showing significant damage on EBPM after chemotherapy (8). There was qualitative research that HER2 was crucial for the construction and maintenance in normal brain tissue (16). HER2 had been shown to be overexpressed in human intracranial tumors, such as gliomas, medulloblastomas, and meningiomas (17). Breast cancer with HER2+ had a higher risk of brain metastasis in comparison to those with the HER2- (18). However, the cognitive function impairment of breast cancer survivors with disparate status of HER2 after chemotherapy was still unclear.

Previous studies made known that COMT (rs4680, rs65599, and rs737865) was closely related to cognitive function (19). COMT gene played an important role in memory, executive control, response suppression, reward processing, decision analysis, and other cognitive processes through the regulation of dopaminergic concentration in the human brain $(20,21)$. Small et al. showed that breast cancer survivors who were COMT Val carriers were susceptible to cognitive deficits following chemotherapy (22). Furthermore, our previous studies had found that COMT (rs165599) gene was associated with retrospective memory (RM) in triple-negative breast cancer (TNBC) survivors (23). Recently, we found that COMT (rs737865) was correlated to EBPM in breast cancer patients with different hormonal receptor expression (24). However, the correlation between the chemotherapy-related PM impairment and the COMT polymorphisms in breast cancer patients with the disparate status of HER2 had not yet been illustrated.

In the current research, we attempt to survey the chemotherapy-related PM impairment in breast cancer survivors with different HER2 and clear cut the genetic features of COMT polymorphisms on CRCI in breast cancer patients with the disparate status of HER2 (HER2-, HER2+).

\section{MATERIALS AND METHODS}

\section{Participants}

A total of 221 breast cancer patients, who were recruited from 2014 to 2017 in the Department of Oncology, the Affiliated Second Hospital of Anhui Medical University, were assigned to HER2 - (103 cases) or HER2+ group (118 cases).

The Research Ethics Committee of the Second Affiliated Hospital of Anhui Medical University, China, approved the research. Written informed consents were obtained from all participants before the research was conducted. Epidemiological data and blood samples were collected in accordance with ethical regulations.

All participants had exceeded 5 years of education and were all right handed. Inclusion criteria were as follows: (1) breast cancer was defined by immunohistochemical and pathological diagnosis and that positive of Her-2 was recorded as standard immunohistochemistry $3+$ or ISH positive; (2) adriamycin, paclitaxel, cyclophosphamide, and fluorouracil were applied by standard chemotherapy regimen or combined with Herceptintargeted therapy, based on chemotherapy, but no hormone therapy; (3) age and pathological type were not limited; (4) the participant could carry out normal daily activities, with Karnofsky performance status scale (KPS) scores $\geq 80$; and (5) there are no communication barriers and could proceed with normal language communication. Exclusion criteria were as follows: (1) a history of radiotherapy and endocrine therapy; 
(2) advanced cachexia; (3) metastatic encephaloma according to brain imaging examination; (4) anxiety, depression, paranoia, and other mental disorders; (5) medical history of alcohol or psychotropic drug dependence; and (6) clinical diagnose of dementia.

\section{General Assessment of Cognitive}

In accordance with the upward grouping of breast cancer survivors, a battery of cognitive tasks were performed within 1 month before chemotherapy and after six cycles of standard postoperative adjuvant chemotherapy. Mini-mental state examination (MMSE) was used to evaluate general cognitive functioning and the degree of intellect, containing seven aspects, presenting in time orientation, place orientation, immediate memory, attention and computational power, delayed memory, language, and visual space. The verbal fluency test (VFT) was reflected in the patient's ability to invoke certain kinds of things from the memory base, mainly measuring the ability of spontaneous language movement, where participants were required to speak out as many targets as they could remember in $1 \mathrm{~min}$. The digit span test (DST) was applied to test patients' short-term memory, including in order and inverted order tests. Participants were required to reiterate the numbers by reading them out to the researchers. The total score corresponded to the number of the last correct character string retelling from the subjects.

\section{Event-Based Prospective Memory Task}

On each card of the 32 cards used, 12 high-frequency Chinese words were printed, of which 10 of 12 words belong to the first category (large category) and the spare two words belong to the second category (animal category). In the learning stage, the participants were required to say the two words pertaining to the small category that differed from the other 10 words on each card. The first two cards were for learning; the first card did not contain the target word, while the second one did. According to the instructions from the experimenter before the test, the target events for the PM task occurred on the 2th (exercise card), 6th, 11th, 16th, 21th, 25th, and 31th card, and each correct score was 1 point; all had 6 points. When the selected word was the target word (animal category), the participants were instructed to tap at the table. At the termination of card selection, they completed another task, that is, let the participants remember to leave their contact number (counted as 2 points). The highest scores of the event-based prospective memory (EBPM) tasks were 8 points.

\section{Time-Based Prospective Memory Task}

On each card of the 100 cards, 12 different two-digit numbers were printed. In the learning stage, participants were required to name the smallest and the largest numbers on each card. According to the instructions from experimenters before the task, when a specific goal time (i.e., at the time points of 5, 10, and 15 min after the beginning of the task), the participants were instructed to knock on the table: 2 points were endowed for responding within $10 \mathrm{~s}$ before and $10 \mathrm{~s}$ after each target time, and
1 point was endowed for responding within $30 \mathrm{~s}$ before and $30 \mathrm{~s}$ after each target time, with a topmost score of 6 points. The participant was told that the time can be checked through the clock placed $1 \mathrm{~m}$ away behind the subject's right shoulder. The clock was set to 0:0:0 at the beginning of the experiment, and the task was stopped when the clock indicated $17 \mathrm{~min}$. The maximum score of time-based prospective memory (TBPM) was 6 points.

\section{Genotyping}

The peripheral blood $(3-5 \mathrm{ml})$ of the subject was sampled into the sodium citrate anticoagulation blood tubes and reserved in the refrigerator at $-80^{\circ} \mathrm{C}$. Genomic DNA was picked up from the peripheral blood with blood genomic DNA Qiagen Kit (Shanghai Genesky Biotechnology Co., Ltd., http://biotech.geneskies.com), operated according to the instructions, and the extracted DNA was stored at $-20^{\circ} \mathrm{C}$. Genotyping was completed by Shanghai Genesky Biotechnology Co., Ltd. (Shanghai, China), utilizing the improved multiplex ligase detection reaction (iMLDR) technology. Different fluorescently labeled allele-specific oligonucleotide probe pairs were used to identify each SNP allele with high specificity. Nonspecific sequences of different lengths were introduced into the end of the ligation probe, and the ligation products were obtained by ligase chain reaction corresponding to the site. Then, the ligation products were amplified by PCR with fluorescent-labeled universal primers. The PCR-amplified products were separated by fluorescence capillary electrophoresis. Finally, GeneMapper 4.1 (Applied Biosystems, USA) was used to analyze the electropherogram; the genotyping success rate of each SNP locus was obtained. A sample accounting for $10 \%$ of the total DNA samples was randomly selected for duplicate tests for quality control.

\section{Statistical Analysis}

Statistical analysis was performed with a one-way ANOVA using SPSS software package (version 22.0, http://spss.en. softonic.com/; Chicago, IL, USA).The basic clinical data and neuropsychological tasks scores were compared between HER2and HER2+ group. The two independent samples t-test and Mann-Whitney U-test were performed, respectively, for normal and non-normal distribution in continuous variable data. All results are presented in the forms of mean \pm standard deviation (SD). Hardy-Weinberg equilibrium (HEW) was applied to analyze whether the distribution of genotype frequency of SNP loci conforms the genetic balance in two groups. In addition, the chi-square $\left(\chi^{2}\right)$ test was used to analyze the differences in alleles, genotype frequency, and other taxonomic variables between the two groups. Logistic regression was reported as the relative risk, odds ratio (OR), and 95\% confidence interval (CI), evaluating the susceptible factors of cognitive impairment; a general genetic model (co-dominant, dominant, recessive, and additive models) to single SNP construes was covered, rectifying age, KPS, chemotherapy regimen, level of education, and pathological pattern. Binary logistic regression was applied to analyze the associations between COMT (rs165599 and rs737865) polymorphism and CRCI. A one-way ANOVA was used to 
analyze the cognitive differences among different genotypes and genetic model (dominant and recessive models). All statistical results were two-tailed probability proofs, and the statistically significant standard was defined at $p<0.05$.

\section{RESULTS}

\section{The Basic Clinical Data for Research Objects}

Table 1 has a total of 221 patients conformed to the inclusion criteria; among them, HER2- group included 103 patients, and HER2+ group included 118 patients. There was no striking difference in age (49.02 \pm 10.95 vs. $48.56 \pm 10.45)$, level of education (10.09 \pm 3.63 vs. $10.10 \pm 3.67)$, and KPS (82.91 \pm 8.12 vs. $84.07 \pm 7.76$ ). Similarly, no significant differences were found for pathological patterns and cancer stages. In the HER2- group, 95 breast cancer patients were discriminated as non-special-type invasive ductal carcinoma (IDO-NOS), 3 breast cancer patients were discriminated as special-type invasive ductal carcinoma (IDO-S), and 5 patients were discriminated as microinvasive carcinoma (MIC). Similarly, in the HER2+ group, 112 breast cancer patients were discriminated as IDO-NOS, 1 breast cancer patients was identified as carcinoma in situ (CIS), and 5 breast cancer patient was identified as MIC. The percentages of stage I (3.9\% and 5.9\%, respectively) and stage II (52.4\% and $48.3 \%$, respectively) were found in breast cancer patients for the two groups. There was significant difference in chemotherapy regimen between the two groups $\left(\chi^{2}=32.101, p<0.01\right)$. The utilization rate of Trastuzumab accounted for about $23.7 \%$ in the HER2+ group.

\section{General Assessment of Cognitive, EBPM, and TBPM Scores: Before and After Chemotherapy}

Table 2 reveals that the MMSE was significantly lessened to $26.67 \pm 1.64$ after chemotherapy in comparison to that before chemotherapy $(27.21 \pm 1.59, p<0.01)$. DST and VFT scores were also strikingly lessened from before $(6.21 \pm 0.71$ and $11.43 \pm 1.53$, respectively) to after $(5.79 \pm 0.99, p<0.01$ and $9.93 \pm 2.14, p<$ 0.01 , respectively) chemotherapy. The EBPM and TBPM scores were significantly decreased after chemotherapy and manifested as $2.72 \pm 0.98$ vs. $1.84 \pm 1.06(p<0.01), 4.95 \pm 1.03$ vs. $4.75 \pm 0.92$ $(p<0.05)$ and had a significant difference.

\section{General Assessment of Cognitive, EBPM, and TBPM Scores: After Chemotherapy}

Table 3 indicates the MMSE and TBPM scores of breast cancer patients in the HER2+ group after chemotherapy was raised (HER2-: $26.43 \pm 1.65$ vs. $4.62 \pm 0.83$; HER2+: $26.89 \pm 1.60$ vs.4.86 $\pm 0.98, \mathrm{p}<0.05)$. Significantly, the DST, VFT, and EBPM were raised in the HER2+ group and manifested as DST of $5.44 \pm$ 0.97 vs. $6.09 \pm 0.90$, VFT of $9.10 \pm 2.14$ vs. $10.65 \pm 1.86$, and EBPM of $1.29 \pm 1.13$ vs. $2.32 \pm 0.72$ and had a significant difference $(p<0.01)$.

\section{The Unit SNP Loci Analytical Results}

The three SNPs of the COMT gene all conformed to HardyWeinberg equilibrium (HWE) for the two groups $(p>0.05)$. It indicated the SNP loci gene frequency distribution we chose from large randomly mating population.

Table 4 shows that the allelic distribution of COMT (rs165599 G vs. A; rs737865 A vs. G) were strikingly different between HER2- and HER2+ survivors $(p=0.045, p=0.012$, respectively). In Table 5, COMT rs165599 (co-dominant model: $\chi^{2}=6.909, p=0.032$; dominant model: $\left.\chi^{2}=6.042, p=0.014\right)$ and rs737865 (co-dominant model: $\chi^{2}=10.993, p=0.004$; dominant model: $\chi^{2}=4.766, p=0.029$; recessive model: $\chi^{2}=7.418, p=$ $0.006)$ genotypic frequency distribution acted out strikingly different. Besides, logistic regression analysis results revealed that COMT rs165599 G/A genotypes [rectified, OR = 0.399, CI $(95 \%)=0.174-0.918, p=0.031]$ had strikingly reduced occurrences of expanding cognitive descend than the patients with G/G. For the genetic model, the dominant model of rs165599 with G/A and A/A genotype [rectified, OR $=2.21$, CI $(95 \%)=1.156-4.225, p=0.016]$ could reduce the risk of cognitive decline. The A/G and $\mathrm{G} / \mathrm{G}$ [rectified, $\mathrm{OR}=0.178, \mathrm{CI}$ $(95 \%)=0.054-0.579, p=0.004 ;$ OR $=0.285$, CI $(95 \%)=0.086-$ $0.947, p=0.040$, respectively] genotype of the COMT rs737865 had strikingly lower odds of expanding cognitive descend than the patients with the A/A genotype. The rs737865 was discovered to strikingly enhance the venture of CRCI in the dominant model [rectified, OR $=1.999, \mathrm{CI}(95 \%)=1.139-3.509, p=0.016]$ and recessive model $[$ rectified, $\mathrm{OR}=4.595$, CI $(95 \%)=1.453-14.532$, $p=0.009$ ]. When comparing the addictive models [rectified, OR $=0.769, \mathrm{CI}(95 \%)=0.450-1.408, p=0.433]$, no significant correlations were established for COMT rs737865. There was no statistically striking difference in the locus of COMT rs4680 between the HER2- and HER2+ group.

\section{The Correlation Analysis Between COMT (rs165599 and rs737865) Gene Polymorphisms and $\mathrm{CRCl}$}

As Table 6 shows, the A/A genotype carriers of COMT rs165599 showed distinctly elevated scores on TBPM $(4.94 \pm 0.75$ vs. $4.42 \pm 0.71, p<0.05)$ than $\mathrm{G} / \mathrm{G}$ carriers in breast cancer patients with disparate status of HER2. Similarly, the G/G and A/G genotype carriers of COMT rs737865 represented higher scores on MMSE (HOM: $24.50 \pm 2.38$ vs. $26.88 \pm 1.26, p<0.01$; HET: $25.87 \pm 1.85$ vs. $26.88 \pm 1.26, p<0.01$, respectively) tests and TBPM (dominant model: $4.88 \pm 0.71$ vs. $4.45 \pm 0.87, p<0.01$; HET: $4.89 \pm 0.69$ vs. $4.45 \pm 0.87, p<0.01$, respectively) tests than A/A carriers.

\section{DISCUSSION}

The results of the current study revealed that, first, breast cancer survivors after chemotherapy had memory impairment on EBPM and TBPM compared to that before chemotherapy. Second, breast cancer patients with HER2- have poorer MMSE, DST, VFT, TBPM, and EBPM scores after 
TABLE 1 | The basic clinical dates of breast cancer patients with HER2- and HER2+.

\begin{tabular}{|c|c|c|c|}
\hline \multirow[t]{2}{*}{ Items } & & \multicolumn{2}{|c|}{ Groups } \\
\hline & & $A(n=103)$ & $B(n=118)$ \\
\hline Age (mean $\pm S D$, year) & & $49.02 \pm 10.95$ & $48.56 \pm 10.45$ \\
\hline Education (mean $\pm \mathrm{SD}$, year) & & $10.09 \pm 3.63$ & $10.10 \pm 3.67$ \\
\hline $\mathrm{KPS}$ (mean $\pm \mathrm{SD}$, year) & & $82.91 \pm 8.12$ & $84.07 \pm 7.76$ \\
\hline \multirow[t]{4}{*}{ Pathological patterns (\%) } & IDC-NOS & 95 (92.2\%) & $112(94.9 \%)$ \\
\hline & IDC-S & 3 (2.9\%) & 0 \\
\hline & ClS & 0 & 1 (0.8\%) \\
\hline & MIC & 5 (4.9\%) & 5 (4.2\%) \\
\hline \multirow[t]{4}{*}{ Stages (\%) } & 1 & 4 (3.9\%) & 7 (5.9\%) \\
\hline & $\|$ & 54 (52.4\%) & 57 (48.3\%) \\
\hline & III & 22 (21.4\%) & $18(15.3 \%)$ \\
\hline & IV & 23 (22.3\%) & 36 (30.5\%) \\
\hline \multirow[t]{4}{*}{ Chemotherapy regimen } & PTX & $6(5.8 \%)$ & $13(11.0 \%)^{\star \star}$ \\
\hline & Trastuzumab + chemotherapy & 0 & $19(16.1 \%)$ \\
\hline & ADM & 22 (21.4\%) & 28 (23.7\%) \\
\hline & PTX+ADM & 75 (72.8\%) & $58(49.2 \%)$ \\
\hline
\end{tabular}

${ }^{* *}<0.01$.

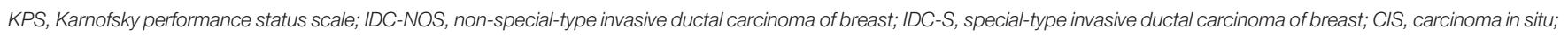
MIC, microinvasive carcinoma; PTX, pacliaxcl; ADM, adriamycin.

chemotherapy than that of patients with HER+. Third, there were significant differences on genotypes about COMT (rs165599 and rs737865) between HER- and HER+ groups; the A/A carriers of COMT rs165599 and the G/G and A/G carriers of COMT rs737865 performed more poorly than COMT (G/G, A/A, respectively) carriers on tests of TBPM in breast cancer patients with HER2-, and the COMT polymorphism may be an underlying genetic factor for the enhancement of the venture to chemotherapy-related PM impairment in breast cancer patients with disparate status of HER2. The results of this study are innovative in that they represent the first demonstration of a link between a risk factor for CRCI and COMT genotype in breast cancer patients with the disparate status of HER2.

Cancer patients will have a series of cognitive changes after chemotherapy, among which memory impairment was one of the main performances (25). Kanaskie et al. (26) believes that the changes in cognitive function are the side effects after chemotherapy for some breast cancer survivors, which include subtle changes in memory, attention, and executive function. Ibrahim et al. (27) found that Taxane-based cognitive impairment is more common in the areas of attention, executive function, and depression, and visual memory in

TABLE 2 | General assessment of cognitive before and after chemotherapy.

\begin{tabular}{lrc}
\hline \multirow{2}{*}{ Task } & \multicolumn{2}{c}{ Mean \pm SD } \\
\cline { 2 - 3 } & Before chemotherapy $(\mathbf{n = 2 2 1 )}$ & After chemotherapy $(\mathbf{n}=\mathbf{2 2 1})$ \\
\hline MMSE & $27.21 \pm 1.59$ & $26.67 \pm 1.64^{\star \star}$ \\
DST & $6.21 \pm 0.71$ & $5.79 \pm 0.99^{\star \star}$ \\
VFT & $11.43 \pm 1.53$ & $9.93 \pm 2.14^{\star \star}$ \\
EBPM & $2.72 \pm 0.98$ & $1.84 \pm 1.06^{\star *}$ \\
TBPM & $4.95 \pm 1.03$ & $4.75 \pm 0.92^{\star}$ \\
\hline
\end{tabular}

${ }^{*} p<0.05,{ }^{* *} p<0.01$

MMSE, mini-mental state examination; DST, digit span test; VFT, verbal fluency test; EBPM, event-based prospective memory; TBPM, time-based prospective memory. breast cancer patients at 6 months or more after treatment. Andryszak et al. found that anthracycline-based adjuvant chemotherapy (AC) was associated with delayed memory deficits after chemotherapy, and about $19 \%$ of breast cancer patients deteriorated after treatment (28). Our previous study found that breast cancer patients mainly present with PM impairment after chemotherapy, especially EBPM deficits (9). Further research found that breast cancer patients with ER-/PRperformed worse on EBPM than those with $\mathrm{ER}+/ \mathrm{PR}+$ after chemotherapy (8). In this study, 221 breast cancer patients were found to have a decline in cognitive function following chemotherapy, and in breast cancer patients with disparate expression of HER2, there exists an obvious difference in EBPM and TBPM after chemotherapy.

HER2 is a proto-oncogene, which can lead to resistance to tumor cells apoptosis and the proliferation tumor blood vessels and lymphatic vessels (29). HER2 was a prognostic factor, which was closely related to recurrence-free survival and overall survival; approximately $18-30 \%$ breast cancer patients shows high expression of HER2 (30). HER2-positive breast cancer patients can be assigned to luminal B (endocrine therapy responsive) or HER2 enriched (endocrine therapy unresponsive), according to their molecular subtypes (31). The combination of trastuzumab (the most widely used anti-HER2 drug) with chemotherapy resulted in significant improvement in the poor prognosis of early HER2+ breast cancer patients and reduced the recurrence risk and the mortality (32). With the application of trastuzumab, about $85 \%$ of HER $2+$ breast cancer patients were expected to survive for at least 10 years, and the prognosis of these patients has improved dramatically (33). Trastuzumab can prolong the survival time of breast cancer patients, but the research on the effect of anti-HER2 therapy on cognitive function is very rare and controversial, and the findings on the correlation between HER2 status and cognitive deficits are full of contradictions. Some studies showed that there was no correlation between cancer HER2 status and pre-adjuvant 
TABLE 3 | General assessment of cognitive in HER2- and HER2+ groups after chemotherapy.

\begin{tabular}{lrr}
\hline \multirow{2}{*}{ Task } & \multicolumn{2}{c}{ Groups (mean \pm SD) } \\
\cline { 2 - 3 } & Her2- $(\mathbf{n}=\mathbf{1 0 3})$ & Her2+ $(\mathbf{n}=\mathbf{1 1 8})$ \\
\hline MMSE & $26.43 \pm 1.65$ & $26.89 \pm 1.60^{\star}$ \\
DST & $5.44 \pm 0.97$ & $6.09 \pm 0.90^{\star \star}$ \\
VFT & $9.10 \pm 2.14$ & $10.65 \pm 1.86^{\star \star}$ \\
EBPM & $1.29 \pm 1.13$ & $2.32 \pm 0.72^{\star \star}$ \\
TBPM & $4.62 \pm 0.83$ & $4.86 \pm 0.98^{\star}$ \\
\hline
\end{tabular}

${ }^{*} p<0.05,{ }^{* *} p<0.01$.

MMSE, mini-mental state; DST, digit span test; VFT, verbal fluency test; EBPM, eventbased prospective memory; TBPM, time-based prospective memory.

therapy cognitive impairment in elderly breast cancer ( $>65$ years of age) (34). On the contrary, Koleck et al. (35) found that the HER2-positive breast cancer patients were more likely to get poorer verbal, visual, and visual working memory performance compared to HER2-negative patients before adjuvant chemotherapy. One study found that the slight to significant deterioration of cognitive function was reported in breast cancer treatment following chemotherapy regimens containing trastuzumab (36). Lee et al. identified that chemo-brain was induced after trastuzumab treatment in an HER2-positive gastric cancer model, and atorvastatin could improve the cognitive impairment caused by trastuzumab (37). However, there were also findings indicating that the administration of subcutaneous trastuzumab can reduce the symptoms of nausea and vomiting caused by chemotherapy and had no negative impact on healthrelated quality of life (38). The incidence of suspected mild cognitive impairment was $28.6 \%$ in the trastuzumab plus chemotherapy group. It showed slightly better cognitive function than that with trastuzumab mono-therapy in HER2+ breast cancer (39). Until now, there is no report regarding CRCI in breast cancer with anti-HER2 therapy. In this study, breast cancer patients with HER2- have a more significant damage on neuropsychological tasks than patients with HER2+. This may be due to the improvement of cognitive function in patients with trastuzumab combined with chemotherapy. In the HER2group, TNBC patients accounted 78.64\%; the CRCI of this group was strikingly higher than that of the HER2+ group, which was consistent with our previous research (23).
The COMT gene was expressed throughout the brain, and its translation products played a key role in clearing catecholamines, such as dopamine, epinephrine, and norepinephrine (40). The expression level and product of COMT gene are affected by many factors. Breast cancer is a tumor closely associated with estrogen, and estrogen could downregulate the level of COMT gene, decreasing the activity of COMT enzyme (41). It has been found that estrogen inhibits COMT gene transcription via promoter reporter gene (42). Estrogen enhanced the promoters of DNMT3B, MBD2, and HDAC1 in breast cancer cells and reduced COMT transcription, resulting in increased DNA oxidative damage (43). Catecholestrogens were estradiol and estrone metabolites produced in breast cancer cells, and its derivatives could initiate estrogen receptor-mediated processes (44). The expression of COMT mRNA and protein was decreased by the proinflammatory cytokine tumor necrosis factor alpha (TNF $\alpha$ ) in astrocytes, and neuroinflammation could be found in the recovery phase (45). There were at least eight different SNPs loci obtained on COMT gene, among which Val158Met locus had been studied the most frequently (46). COMT polymorphisms are manifested as a valine (Val or $\mathrm{G}$ ) and methionine (Met or A) mutation at codon 158. The activity of the COMT enzyme with the Met carriers was three- to fourfold reduced than that with the Val carriers, increasing the dopaminergic concentration of synapses in the human brain (47). McIntosh et al. (48) found that the anterior cingulate cortex of Val homozygous carriers was significantly smaller than that of met carriers in schizophrenic patients; the altered brain structure could lead to cognitive impairment. COMT gene was widely expressed in the hippocampus and was associated with memory function $(19,49)$. Correa et al. (50) found that COMT SNPs were strikingly associated with attention, executive functions, and memory scores in patients with brain tumor. Matsuzaka et al. (51) showed the relationship between the two SNPs of the COMT (rs165599 and rs737865) and working memory; the cognition in schizophrenia patients may be modulated by COMT. Compared with healthy controls, breast cancer patients receiving chemotherapy had slower treatment speed and poorer executive function, while apolipoprotein E (APOE) and COMT gene polymorphisms were associated with cognitive impairment (52). Our previous research findings indicated that

TABLE 4 | Information about three genotyped SNPs loci of COMT gene in HER2- and HER2+ groups .

\begin{tabular}{|c|c|c|c|}
\hline \multirow[t]{2}{*}{ SNP } & \multicolumn{3}{|c|}{ COMT } \\
\hline & rs4680 & rs165599 & rs737865 \\
\hline CHR & 22 & 22 & 22 \\
\hline Allele position & 19951271 & 19956781 & 19930121 \\
\hline Ref allele & G & G & A \\
\hline Alt allele & $A$ & A & $\mathrm{G}$ \\
\hline MAF & 0.233 & 0.422 & 0.226 \\
\hline$P$ for HWE & 0.279 & 0.227 & 0.261 \\
\hline$p^{*}$ & 0.648 & 0.045 & 0.012 \\
\hline
\end{tabular}

SNP, single-nucleotide polymorphism; CHR, chromosome; Ref allele, loci alleles on the reference sequence; Alt allele, the other allele on the loci; MAF, minor allele frequency (data from 1000 Genomes); HWE, Hardy-Weinberg equilibrium, p-value for HWE in two groups.

${ }^{*} p$-value for allele frequency differences between two groups. 
TABLE 5 | Genotype frequencies of SNPs of the COMT (rs4680, rs165599, and rs737865) genes between two groups.

\begin{tabular}{|c|c|c|c|c|c|c|c|}
\hline SNP & Model & Genotype & Her2 (-) & Her2 (+) & $p^{\mathrm{a}}\left(\chi^{2}\right)$ & \multicolumn{2}{|c|}{ Logistic regression } \\
\hline \multirow[t]{5}{*}{ rs4680 } & Co-dominant & $G / G$ & 63 & 65 & & - & - \\
\hline & & $\mathrm{A} / \mathrm{A}$ & 8 & 9 & & $1.597(0.524-4.865)$ & 0.410 \\
\hline & Dominant & $\mathrm{G} / \mathrm{A}+\mathrm{A} / \mathrm{A}$ & 40 & 53 & 0.361 & $1.233(0.706-2.153)$ & 0.461 \\
\hline & & $\mathrm{G} / \mathrm{G}$ & 63 & 65 & & & \\
\hline & Addictive & - & - & - & - & $0.732(0.409-1.308)$ & 0.292 \\
\hline \multirow[t]{5}{*}{ rs165599 } & Co-dominant & $\mathrm{G} / \mathrm{G}$ & 33 & 21 & & - & - \\
\hline & & $\mathrm{G} / \mathrm{A}$ & 53 & 67 & 0.032 & $0.399(0.174-0.918)$ & 0.031 \\
\hline & & $\mathrm{A} / \mathrm{A}$ & 17 & 30 & & $0.84(0.407-1.736)$ & 0.638 \\
\hline & Dominant & $\mathrm{G} / \mathrm{A}+\mathrm{A} / \mathrm{A}$ & 70 & 97 & 0.014 & $2.21(1.156-4.225)$ & 0.016 \\
\hline & & $\mathrm{G} / \mathrm{G}$ & 33 & 21 & & & \\
\hline \multirow{7}{*}{ rs737865 } & & $\mathrm{A} / \mathrm{G}$ & 38 & 47 & 0.004 & $0.178(0.054-0.579)$ & 0.004 \\
\hline & & $\mathrm{G} / \mathrm{G}$ & 4 & 19 & & $0.285(0.086-0.947)$ & 0.040 \\
\hline & Dominant & $\mathrm{A} / \mathrm{G}+\mathrm{G} / \mathrm{G}$ & 42 & 66 & 0.029 & 1.999 (1.139-3.509) & 0.016 \\
\hline & & $\mathrm{A} / \mathrm{A}$ & 60 & 52 & & & \\
\hline & Recessive & $\mathrm{G} / \mathrm{G}$ & 4 & 19 & 0.006 & $4.595(1.453-14.532)$ & 0.009 \\
\hline & & $\mathrm{A} / \mathrm{A}+\mathrm{A} / \mathrm{G}$ & 98 & 99 & & & \\
\hline & Addictive & - & - & - & - & 0.769 (0.450-1.408) & 0.433 \\
\hline
\end{tabular}

${ }^{a}$ The $\chi^{2}$ test of $p$-values for SNP polymorphisms distribution differences between Her2(-) and Her2(+) group.

${ }^{b} \mathrm{p}$-value for logistic regression analysis; odds ratio (the OR); $95 \%$ confidence interval ( $\left.95 \% \mathrm{Cl}\right)$; models: various genetic models that were defined as 1 (MM + Mm) versus 0 (mm) for dominant; $1(\mathrm{~mm})$ versus $0(\mathrm{MM}+\mathrm{Mm})$ for recessive; and $0(\mathrm{~mm})$ versus $1(\mathrm{Mm})$ versus $2(\mathrm{MM})$ for additive and co-dominant ( $\mathrm{M}$ and $\mathrm{m}$ represent major and minor alleles, respectively).

COMT (rs165599) was a risk-related genetic factor influencing CRCI in TNBC patients (23). Further study found that COMT (rs737865) was correlated with EBPM damage following chemotherapy in breast cancer with different expressions of hormone receptor (24). In this study, the A/A genotype carriers of COMT (rs165599) and G/G genotype carriers of
COMT (rs737865) had higher scores on TBPM after chemotherapy and were genetic risks for CRCI in breast cancer with disparate expression of HER2.

Finally, limitations of this research should be recognized. First, this research only compared the changes in cognitive function in breast cancer patients with disparate expressions of

TABLE 6 | Comparison for neuropsychological performance of COMT (rs165599 and rs737865) genotypes and genetic model.

\begin{tabular}{|c|c|c|c|c|c|c|c|c|}
\hline \multirow{3}{*}{$\begin{array}{l}\text { rs165599 } \\
\text { MMSE }\end{array}$} & \multicolumn{2}{|c|}{ Dominant } & \multicolumn{2}{|c|}{ Recessive } & \multicolumn{2}{|c|}{ HOM } & \multicolumn{2}{|c|}{ HET } \\
\hline & \multicolumn{2}{|c|}{$G / A+A / A$ vs. $G / G$} & \multicolumn{2}{|c|}{ A/A vs. $G / G+G / A$} & \multicolumn{2}{|c|}{ A/A vs. $G / G$} & \multicolumn{2}{|c|}{ G/A vs. $G / G$} \\
\hline & $26.27 \pm 1.72$ & $26.76 \pm 1.48$ & $26.29 \pm 1.65$ & $26.45 \pm 1.66$ & $26.29 \pm 1.65$ & $26.76 \pm 1.48$ & $26.26 \pm 1.76$ & $26.76 \pm 1.48$ \\
\hline DST & $5.42 \pm 0.97$ & $5.49 \pm 0.98$ & $5.56 \pm 1.03$ & $5.42 \pm 0.96$ & $5.56 \pm 1.03$ & $5.49 \pm 0.98$ & $5.38 \pm 0.95$ & $5.49 \pm 0.98$ \\
\hline VFT & $8.87 \pm 2.14$ & $9.58 \pm 2.09$ & $9.35 \pm 2.03$ & $9.05 \pm 2.17$ & $9.35 \pm 2.03$ & $9.58 \pm 2.09$ & $8.72 \pm 2.17$ & $9.58 \pm 2.09$ \\
\hline EBPM & $1.34 \pm 1.17$ & $1.18 \pm 1.04$ & $1.71 \pm 1.36$ & $1.21 \pm 1.06$ & $1.71 \pm 1.36$ & $1.18 \pm 1.04$ & $1.23 \pm 1.09$ & $1.18 \pm 1.04$ \\
\hline TBPM & $4.71 \pm 0.87$ & $4.42 \pm 0.71$ & $4.94 \pm 0.75$ & $4.56 \pm 0.84$ & $4.94 \pm 0.75$ & $4.42 \pm 0.71^{\star}$ & $4.64 \pm 0.90$ & $4.42 \pm 0.71$ \\
\hline rs737865 & $\begin{array}{r}\text { Do } \\
A / G+G\end{array}$ & $\begin{array}{l}\text { nant } \\
\text { vs. A/A }\end{array}$ & $\begin{array}{r}\text { Rec } \\
\text { G/G vs }\end{array}$ & $\begin{array}{l}\text { sive } \\
/ \mathrm{A}+\mathrm{A} / \mathrm{G}\end{array}$ & $\mathrm{G} / \mathrm{G}$ & $M$ & A/G & $\mathrm{A} / \mathrm{A}$ \\
\hline MMSE & $25.74 \pm 1.91$ & $26.88 \pm 1.26^{\star \star}$ & $24.50 \pm 2.38$ & $26.49 \pm 1.59^{\star}$ & $24.50 \pm 2.38$ & $26.88 \pm 1.26^{\star \star}$ & $25.87 \pm 1.85$ & $26.88 \pm 1.26^{\star \star}$ \\
\hline DST & $5.52 \pm 1.04$ & $5.39 \pm 0.92$ & $5.25 \pm 0.96$ & $5.45 \pm 0.97$ & $5.25 \pm 0.96$ & $5.39 \pm 0.92$ & $5.55 \pm 1.06$ & $5.39 \pm 0.92$ \\
\hline VFT & $8.93 \pm 1.92$ & $9.25 \pm 2.29$ & $8.75 \pm 2.75$ & $9.13 \pm 2.13$ & $8.75 \pm 2.75$ & $9.25 \pm 2.29$ & $8.89 \pm 1.86$ & $9.25 \pm 2.29$ \\
\hline EBPM & $1.12 \pm 1.09$ & $1.43 \pm 1.14$ & $1.75 \pm 1.50$ & $1.29 \pm 1.11$ & $1.75 \pm 1.50$ & $1.43 \pm 1.14$ & $1.05 \pm 1.04$ & $1.43 \pm 1.14$ \\
\hline TBPM & $4.88 \pm 0.71$ & $4.45 \pm 0.87^{\star \star}$ & $4.75 \pm 0.96$ & $4.62 \pm 0.83$ & $4.75 \pm 0.96$ & $4.45 \pm 0.87$ & $4.89 \pm 0.69$ & $4.45 \pm 0.87^{\star \star}$ \\
\hline
\end{tabular}

${ }^{*} p<0.05 ;{ }^{* *} p<0.01$

MMSE, mini-mental state; DST, digit span test; VFT, verbal fluency test; EBPM, event-based prospective memory; TBPM, time-based prospective memory.

Models: Various genetic models that were defined as $1(\mathrm{MM}+\mathrm{Mm})$ versus $\mathrm{O}(\mathrm{mm})$ for dominant; 1 ( $\mathrm{mm})$ versus $\mathrm{O}(\mathrm{MM}+\mathrm{Mm})$ for recessive; homozygote $(\mathrm{HOM})$; heterozygote $(\mathrm{HET})$. 
HER2 before and after chemotherapy, lacking a healthy control group. Second, follow-up study was lacking. Cognitive impairments following chemotherapy may change in the later follow-up period. Thus, further research is needed. Third, the results were subjective memory impairment; further objective cognitive tests need to be clarified in future research. Fourth are the impacts of chemotherapy regimens. If some regimens had negative effects on cognitive function, but others did not, the effects of the former would be diluted and undetectable. Finally, the sample size in this research was small, and the numbers of breast cancer patients were scarce, therefore needing further supplement.

In a word, our study preliminarily found some differences in chemotherapy-related PM impairment and genetic polymorphisms in breast cancer patients with the disparate HER2. The heterogeneity of CRCI may be rectified by COMT (rs165599, rs737865) polymorphism, and this rectification may possibly show that COMT polymorphism is a risk leading to a lower memory performance in breast cancer patients with disparate HER2.

\section{CONCLUSION}

In brief, we conducted the discrepancy between chemotherapyrelated PM impairment and genetic polymorphisms in patients with HER2-/+ breast cancer. The consequences indicated that the heterogeneity of CRCI may be regulated by COMT (rs165599 and rs737865), which may affect the CRCI in breast cancer with disparate status of HER2.

\section{REFERENCES}

1. Wang X, Wang C, Guan J, Chen B, Xu L, Chen C. Progress of Breast Cancer Basic Research in China. Int J Biol Sci (2021) 17(8):2069-79. doi: 10.7150/ijbs.60631

2. Fan L, Strasser-Weippl K, Li JJ, St Louis J, Finkelstein DM, Yu KD, et al. Breast Cancer in China. Lancet Oncol (2014) 15(7):e279-89. doi: 10.1016/ S1470-2045(13)70567-9

3. Harbeck N, Gnant M. Breast Cancer. Lancet (2017) 389(10074):1134-50. doi: 10.1016/S0140-6736(16)31891-8

4. DeSantis CE, Ma J, Gaudet MM, Newman LA, Miller KD, Goding Sauer A, et al. Breast Cancer Statistics, 2019. CA: Cancer J Clin (2019) 69(6):438-51. doi: $10.3322 /$ caac. 21583

5. Keetile NM, Osuch E, Lentoor AG. Chemotherapy-Related Subjective Cognitive Impairment in Breast Cancer Patients in Semi-Rural South Africa. Health SA SA Gesondheid (2021) 26:1605. doi: 10.4102/hsag.v26i0.1605

6. Huehnchen P, van Kampen A, Boehmerle W, Endres M. Cognitive Impairment After Cytotoxic Chemotherapy. Neuro-Oncol Pract (2020) 7 (1):11-21. doi: 10.1093/nop/npz052

7. Hsu YH, Chen VC, Hsieh CC, Weng YP, Hsu YT, Hsiao HP, et al. Subjective and Objective Cognitive Functioning Among Patients With Breast Cancer: Effects of Chemotherapy and Mood Symptoms. Breast Cancer (2021) 28 (1):236-45. doi: 10.1007/s12282-020-01168-y

8. Li W, Gan C, Lv Y, Wang S, Cheng H. Chemotherapy-Induced Prospective Memory Impairment in Breast Cancer Patients With Different Hormone Receptor Expression. Medicine (2017) 96(13):e6514. doi: 10.1097/ MD.0000000000006514

9. Cheng H, Yang Z, Dong B, Chen C, Zhang M, Huang Z, et al. ChemotherapyInduced Prospective Memory Impairment in Patients With Breast Cancer. Psycho-Oncology (2013) 22(10):2391-5. doi: 10.1002/pon.3291

\section{DATA AVAILABILITY STATEMENT}

The datasets presented in this study can be found in online repositories. The names of the repository/repositories and accession number(s) can be found below: https://www.ncbi.nlm.nih.gov/, 111.

\section{ETHICS STATEMENT}

The studies involving human participants were reviewed and approved by the Research Ethics Committee of the Second Affiliated Hospital of Anhui Medical University. The patients/ participants provided their written informed consent to participate in this study.

\section{AUTHOR CONTRIBUTIONS}

WL performed data collection, cognitive tests, EBPM and TBPM task, and blood collection. QZ and YC performed statistical analysis. TC performed data acquisition. HC designed the project and wrote the manuscript. All authors contributed to the article and approved the submitted version.

\section{ACKNOWLEDGMENTS}

This research was supported by the National Natural Science Foundation of China (No. 81872504).

10. de Ruiter MB, Reneman L, Kieffer JM, Oldenburg HSA, Schagen SB. Brain White Matter Microstructure as a Risk Factor for Cognitive Decline After Chemotherapy for Breast Cancer. J Clin Oncol Off J Am Soc Clin Oncol (2021) 39(35):3908-17. doi: 10.1200/JCO.21.00627

11. Runowicz CD, Leach CR, Henry NL, Henry KS, Mackey HT, CowensAlvarado RL, et al. American Cancer Society/American Society of Clinical Oncology Breast Cancer Survivorship Care Guideline. J Clin Oncol Off J Am Soc Clin Oncol (2016) 34(6):611-35. doi: 10.1200/JCO.2015.64.3809

12. Lin SZ, Wu YK, Su YA, Si TM. Prospective Memory in non-Psychotic FirstDegree Relatives of Patients With Schizophrenia: A Meta-Analysis. Neuropsychiatr Dis Treat (2019) 15:1563-71. doi: 10.2147/NDT.S203729

13. Kurozumi S, Katayama A, Shirabe K, Horiguchi J, Rakha EA. Clinicopathological Utility of Human Epidermal Growth Factor Receptor 2 (HER2)-Heterogeneity for Next-Generation Treatments of Triple-Negative Breast Cancer. Oncotarget (2021) 12(22):2302-4. doi: 10.18632/ oncotarget. 28007

14. Li YL, Qin YC, Tang LY, Liao YH, Zhang W, Xie XM, et al. Patient and Care Delays of Breast Cancer in China. Cancer Res Treat (2019) 51(3):1098-106. doi: $10.4143 / \mathrm{crt} .2018 .386$

15. Barton MK. Cognitive Deficits are Usually Mild in Patients With Breast Cancer After Chemotherapy. CA: Cancer J Clin (2013) 63(1):3-4. doi: $10.3322 /$ caac. 21164

16. Waage IS, Vreim I. Torp SH. C-Erbb2/HER2 in Human Gliomas, Medulloblastomas, and Meningiomas: A Minireview. Int J Surg Pathol (2013) 21(6):573-82. doi: 10.1177/1066896913492196

17. Schmid RS, McGrath B, Berechid BE, Boyles B, Marchionni M, Sestan N, et al. Neuregulin 1-Erbb2 Signaling Is Required for the Establishment of Radial Glia and Their Transformation Into Astrocytes in Cerebral Cortex. Proc Natl Acad Sci USA (2003) 100(7):4251-6. doi: 10.1073/pnas.0630496100 
18. Kennecke H, Yerushalmi R, Woods R, Cheang MC, Voduc D, Speers CH, et al. Metastatic Behavior of Breast Cancer Subtypes. J Clin Oncol Off J Am Soc Clin Oncol (2010) 28(20):3271-7. doi: 10.1200/JCO.2009.25.9820

19. Tougu P, Tulviste T, Veidebaum T, Harro J. Schoolchildren's Autobiographical Memory: COMT Gene Val(158)Met Polymorphism Effects on Emotional Content and Quality of First Memories. Cogn Process (2021). doi: 10.1007/s10339-021-01064-z

20. Wing VC, Tang YL, Sacco KA, Cubells JF, George TP. Effect of COMT Val (158)Met Genotype on Nicotine Withdrawal-Related Cognitive Dysfunction in Smokers With and Without Schizophrenia. Schizophr Res (2013) 150(23):602-3. doi: 10.1016/j.schres.2013.09.005

21. Favaro A, Clementi M, Manara R, Bosello R, Forzan M, Bruson A, et al. Catechol-O-Methyltransferase Genotype Modifies Executive Functioning and Prefrontal Functional Connectivity in Women With Anorexia Nervosa. J Psychiatry Neurosci JPN (2013) 38(4):241-8. doi: 10.1503/jpn.120068

22. Small BJ, Rawson KS, Walsh E, Jim HS, Hughes TF, Iser L, et al. Catechol-OMethyltransferase Genotype Modulates Cancer Treatment-Related Cognitive Deficits in Breast Cancer Survivors. Cancer (2011) 117(7):1369-76. doi: 10.1002/cncr.25685

23. Cheng H, Li W, Gan C, Zhang B, Jia Q, Wang K. The COMT (Rs165599) Gene Polymorphism Contributes to Chemotherapy-Induced Cognitive Impairment in Breast Cancer Patients. Am J Trans Res (2016) 8(11):5087-97.

24. Li W, Zhao J, Ding K, Chao HH, Li CR, Cheng H, et al. Catechol-OMethyltransferase Gene Polymorphisms and the Risk of ChemotherapyInduced Prospective Memory Impairment in Breast Cancer Patients With Varying Tumor Hormonal Receptor Expression. Med Sci Monitor Int Med J Exp Clin Res (2020) 26:e923567. doi: 10.12659/MSM.923567

25. Oyovwi MO, Ben-Azu B, Edesiri TP, Victor E, Rotu RA, Ozegbe QEB, et al. Kolaviron Abates Busulfan-Induced Episodic Memory Deficit and Testicular Dysfunction in Rats: The Implications for Neuroendopathobiological Changes During Chemotherapy. Biomed Pharmacother Biomed Pharmacother (2021) 142:112022. doi: 10.1016/j.biopha.2021.112022

26. Kanaskie ML, Loeb SJ. The Experience of Cognitive Change in Women With Breast Cancer Following Chemotherapy. J Cancer Survivorship Res Pract (2015) 9(3):375-87. doi: 10.1007/s11764-014-0387-x

27. Ibrahim EY, Domenicano I, Nyhan K, Elfil M, Mougalian SS, Cartmel B, et al. Cognitive Effects and Depression Associated With Taxane-Based Chemotherapy in Breast Cancer Survivors: A Meta-Analysis. Front Oncol (2021) 11:642382. doi: 10.3389/fonc.2021.642382

28. Andryszak P, Wilkosc M, Zurawski B, Izdebski P. Verbal Memory in Breast Cancer Patients Treated With Chemotherapy With Doxorubicin and Cyclophosphamide. Eur J Cancer Care (2018) 27(1):e12749. doi: 10.1111/ecc.12749

29. Jinga DC, Jinga MR, Miron A, Noditi A, Blidaru A. Pathological Response and Survival After Neoadjuvant Therapy for Her-2 Positive Breast Cancer. Chirurgia (2021) 116(2 Suppl):91-7. doi: 10.21614/rurgia.116.2Suppl.S91

30. Cronin KA, Harlan LC, Dodd KW, Abrams JS, Ballard-Barbash R. Population-Based Estimate of the Prevalence of HER-2 Positive Breast Cancer Tumors for Early Stage Patients in the US. Cancer Invest (2010) 28 (9):963-8. doi: 10.3109/07357907.2010.496759

31. Harbeck N. Insights Into Biology of Luminal HER2 vs. Enriched HER2 Subtypes: Therapeutic Implications. Breast (2015) 24 Suppl 2:S44-8. doi: 10.1016/j.breast.2015.07.011

32. Daniels B, Kiely BE, Houssami N, Lord SJ, Dobbins T, Lu CY, et al. Survival Outcomes for Australian Women Receiving Trastuzumab for HER2-Positive Metastatic Breast Cancer Following (Neo)Adjuvant Trastuzumab: A National Population-Based Observational Study (2006-2014). Br J Cancer (2018) 118 (3):441-7. doi: 10.1038/bjc.2017.405

33. Kreutzfeldt J, Rozeboom B, Dey N, De P. The Trastuzumab Era: Current and Upcoming Targeted HER2+ Breast Cancer Therapies. Am J Cancer Res (2020) 10(4):1045-67.

34. Lange M, Giffard B, Noal S, Rigal O, Kurtz JE, Heutte N, et al. Baseline Cognitive Functions Among Elderly Patients With Localised Breast Cancer. Eur J Cancer (2014) 50(13):2181-9. doi: 10.1016/j.ejca.2014.05.026

35. Koleck TA, Bender CM, Sereika SM, Ryan CM, Ghotkar P, Brufsky AM, et al. Associations Between Pathologic Tumor Features and Preadjuvant Therapy Cognitive Performance in Women Diagnosed With Breast Cancer. Cancer Med (2017) 6(2):339-48. doi: 10.1002/cam4.964

36. Garcia-Sanchez J, Torregrosa MD, Cauli O. Cognitive Functions Under AntiHER2 Targeted Therapy in Cancer Patients: A Scoping Review. Endocrine
Metab Immune Disord Drug Targets (2021) 21(7):1163-70. doi: 10.2174/ 1871530320666200729153009

37. Lee S, Lee HJ, Kang H, Kim EH, Lim YC, Park H, et al. Trastuzumab Induced Chemobrain, Atorvastatin Rescued Chemobrain With Enhanced Anticancer Effect and Without Hair Loss-Side Effect. J Clin Med (2019) 8(2):234. doi: $10.3390 / \mathrm{jcm} 8020234$

38. Syrios J, Pappa E, Volakakis N, Grivas A, Alafis J, Manioudaki S, et al. RealWorld Data on Health-Related Quality of Life Assessment in Patients With Breast Cancer Receiving Subcutaneous Trastuzumab. Breast Cancer Basic Clin Res (2018) 12:1178223418758031. doi: 10.1177/1178223418758031

39. Hagiwara Y, Sawaki M, Uemura Y, Kawahara T, Shimozuma K, Ohashi Y, et al. Impact of Chemotherapy on Cognitive Functioning in Older Patients With HER2-Positive Breast Cancer: A Sub-Study in the RESPECT Trial. Breast Cancer Res Treat (2021) 188(3):675-83. doi: 10.1007/s10549-021-06253-0

40. Bonetti L, Bruzzone SEP, Sedghi NA, Haumann NT, Paunio T, Kantojarvi K, et al. Brain Predictive Coding Processes Are Associated to COMT Gene Val158Met Polymorphism. NeuroImage (2021) 233:117954. doi: 10.1016/j.neuroimage.2021.117954

41. Jiang H. Human Catechol-O-Methyltransferase Down-Regulation by Estradiol. Neuropharmacology (2003) 45(7):1011-8. doi: 10.1016/s00283908(03)00286-7

42. Xie T, Ho SL, Ramsden D. Characterization and Implications of Estrogenic Down-Regulation of Human Catechol-O-Methyltransferase Gene Transcription. Mol Pharmacol (1999) 56(1):31-8. doi: 10.1124/mol.56.1.31

43. Wu Q, Odwin-Dacosta S, Cao S, Yager JD, Tang WY. Estrogen Down Regulates COMT Transcription via Promoter DNA Methylation in Human Breast Cancer Cells. Toxicol Appl Pharmacol (2019) 367:12-22. doi: 10.1016/ j.taap.2019.01.016

44. Schutze N, Vollmer G, Tiemann I, Geiger M, Knuppen R. Catecholestrogens are MCF-7 Cell Estrogen Receptor Agonists. J Steroid Biochem Mol Biol (1993) 46(6):781-9. doi: 10.1016/0960-0760(93)90319-r

45. Tchivileva IE, Nackley AG, Qian L, Wentworth S, Conrad M, Diatchenko LB. Characterization of NF-kB-Mediated Inhibition of Catechol-OMethyltransferase. Mol Pain (2009) 5:13. doi: 10.1186/1744-8069-5-13

46. Caldu X, Ottino-Gonzalez J, Sanchez-Garre C, Hernan I, Tor E, SenderPalacios MJ, et al. Effect of the Catechol-O-Methyltransferase Val (158) Met Polymorphism on Theory of Mind in Obesity. Eur Eating Disord Rev J Eating Disord Assoc (2019) 27(4):401-9. doi: 10.1002/erv.2665

47. Vai B, Riberto M, Poletti S, Bollettini I, Lorenzi C, Colombo C, et al. Catechol-OMethyltransferase Val(108/158)Met Polymorphism Affects Fronto-Limbic Connectivity During Emotional Processing in Bipolar Disorder. Eur Psychiatry J Assoc Eur Psychiatrists (2017) 41:53-9. doi: 10.1016/j.eurpsy.2016.10.002

48. McIntosh AM, Baig BJ, Hall J, Job D, Whalley HC, Lymer GK, et al. Relationship of Catechol-O-Methyltransferase Variants to Brain Structure and Function in a Population at High Risk of Psychosis. Biol Psychiatry (2007) 61(10):1127-34. doi: 10.1016/j.biopsych.2006.05.020

49. Laatikainen LM, Sharp T, Bannerman DM, Harrison PJ, Tunbridge EM. Modulation of Hippocampal Dopamine Metabolism and HippocampalDependent Cognitive Function by Catechol-O-Methyltransferase Inhibition. J Psychopharmacol (2012) 26(12):1561-8. doi: 10.1177/0269881112454228

50. Correa DD, Satagopan J, Cheung K, Arora AK, Kryza-Lacombe M, Xu Y, et al. COMT, BDNF, and DTNBP1 Polymorphisms and Cognitive Functions in Patients With Brain Tumors. Neuro-Oncology (2016) 18(10):1425-33. doi: $10.1093 /$ neuonc/now057

51. Matsuzaka CT, Christofolini D, Ota VK, Gadelha A, Berberian AA, Noto C, et al. Catechol-O-Methyltransferase (COMT) Polymorphisms Modulate Working Memory in Individuals With Schizophrenia and Healthy Controls. Rev Bras Psiquiatria (2017) 39(4):302-8. doi: 10.1590/1516-4446-2016-1987

52. Harrison RA, Rao V, Kesler SR. The Association of Genetic Polymorphisms With Neuroconnectivity in Breast Cancer Patients. Sci Rep (2021) 11(1):6169. doi: 10.1038/s41598-021-85768-4

Conflict of Interest: The authors declare that the research was conducted in the absence of any commercial or financial relationships that could be construed as a potential conflict of interest.

Publisher's Note: All claims expressed in this article are solely those of the authors and do not necessarily represent those of their affiliated organizations, or those of the publisher, the editors and the reviewers. Any product that may be evaluated in 
this article, or claim that may be made by its manufacturer, is not guaranteed or endorsed by the publisher.

Copyright $\odot 2022 \mathrm{Li}$, Zhang, Cai, Chen and Cheng. This is an open-access article distributed under the terms of the Creative Commons Attribution License
(CC BY). The use, distribution or reproduction in other forums is permitted, provided the original author(s) and the copyright owner(s) are credited and that the original publication in this journal is cited, in accordance with accepted academic practice. No use, distribution or reproduction is permitted which does not comply with these terms. 